Annals of Pure and Applied Mathematics

Vol. 17, No. 1, 2018, 85-93

ISSN: 2279-087X (P), 2279-0888(online)

Published on 30 April 2018

www.researchmathsci.org

DOI: http://dx.doi.org/10.22457/apam.v17n1a9

Annals of

Pure and Applied

Mathematics

\title{
A Note on the Lattice of $L$-Closure Operators
}

\author{
Pinky $^{1}$ and T.P.Johnson ${ }^{2}$ \\ ${ }^{1}$ Department of Mathematics, Cochin University of Science and Technology \\ Cochin-682022, India. E-mail: pritammalik90@gmail.com \\ ${ }^{2}$ Applied Science and Humanities Divison, School of Engineering, Cochin University of \\ Science and Technology, Cochin-682022, India. E-mail: tpjohnson@cusat.ac.in \\ ${ }^{1}$ Corresponding author
}

Received 21 March 2018; accepted 28 April 2018

Abstract. In this paper, we investigate the lattice structure of the set of all $L$-closure operators on a given nonempty set $X$ when membership lattice $L$ is a bounded chain. It is proved that in this case, the lattice of all $L$-closure operators is distributive, modular but not atomic and not complemented. The authors disprove certain known theorems on the above lattices and the correct results are provided.

Keyword: $L$-topology, Lattice, Chain, $L$-closure operator.

AMS Mathematics Subject Classification (2010): 03G10, 54A99

\section{Introduction}

In 1965, the concept of fuzzy sets and fuzzy set operations were first introduced by Zadeh in his classical paper [12]. In 1968, Chang [2] applied some basic concepts from general topology to fuzzy sets and developed a theory of fuzzy topological spaces. Closure spaces which is a generalization of topological spaces were introduced by Cech [1] and then studied by many authors. They have extended many topological concepts to closure operators. Fuzzy closure spaces were first studied by Mashhour and Ghanim [7]. Fuzzy closure spaces are generalization of fuzzy topological spaces. The definition of Mashhour and Ghanim is analogue of $\breve{C}$ ech closure spaces. Srivastava et al., [9] have introduced fuzzy closure spaces as analogue of Brikhoff closure spaces. Srivastava and Srivastava [10] have studied the subspace of a fuzzy closure space and introduced the notion of a $\mathrm{T}_{1}$ -fuzzy closure space. Johnson [5] has studied the lattice structure of the set $L(X)$ of all $\breve{C}$ ech fuzzy closure operators on a fixed set $X$ and proved that $L(X)$ is a complete lattice but not complemented. Zhou [11] has introduced the concepts of $L$-closure spaces and the convergence in $L$-closure spaces.

In this paper, we have investigated the lattice structure of the lattice $L C(X)$ of all $L$-closure operators on a given non-empty set $X$ when membership lattice $L$ is a bounded chain. In addition, we have identified the infra $L$-closure operators and their number and established a relation between ultra $L$-topology and ultra $L$-closure operator. 


\section{Pinky and T.P Johnson}

\section{Preliminaries}

Throughout this paper, $X$ stands for a non-empty set, $L$ for a bounded chain with the least element 0 and the greatest element 1 , which is a completely distributive lattice with an order reversing involution ' ' , (i.e. $\forall a, b \in L, a \leq b \Rightarrow a^{\prime} \geq b^{\prime}$ and for every $a \in L, a^{\prime \prime}=a$ ) and

$L^{X}=\{f: f: X \rightarrow L$ is a mapping $\}$. The constant function in $L^{X}$, taking value $\alpha$ is denoted by $\underline{\alpha}$ and $x_{\gamma}$, where $\gamma(\neq 0) \in L$, denotes the $L$ - fuzzy point defined by

$$
x_{\gamma}(y)=\left\{\begin{array}{llc}
\gamma & \text { if } & y=x \\
0 & \text { otherwise }
\end{array} .\right.
$$

Any $f \in L^{X}$ is called as an $L$-subset of $X$ and the complement of $f$, denoted by $f^{\prime}$ is defined by the formula $f^{\prime}(x)=[f(x)]^{\prime}$. The following are some important definition reported in $[3,6]$ :

Definition 2.1. An element of $L$ is called an atom if it is a minimal element of $L \backslash\{0\}$.

Definition 2.2. An element of $L$ is called a dual atom if it is a maximal element of $L \backslash\{1\}$.

Definition 2.3. Let $\delta$ be a nonempty subset of $L^{X}$. We call $\delta$ an L-topologyon $X$, if $\delta$ satisfies the following conditions :

(1) $\underline{0}, \underline{1} \in \delta$.

(2) if $f, g \in \delta$, then $f \wedge g \in \delta$.

(3) if $\delta_{1} \subseteq \delta$, then $\vee_{f \in \delta_{1}} f \in \delta$.

The pair $\left(L^{X}, \delta\right)$ is called an $L$-topological space.

In this paper, we take the definition of $L$-closure operator as a generalization of fuzzy closure operator in [7].

Definition 2.4. A Cech $L$-closure operator on a set $X$ is a function $c: L^{X} \rightarrow L^{X}$ satisfying the following three axioms :

(1) $c(\underline{0})=\underline{0}$.

(2) $f \leq c(f)$ for every $f$ in $L^{X}$.

(3) $c(f \vee g)=c(f) \vee c(g)$ for all $f, g \in L^{X}$.

For convenience, we call it a $L$-closure operator on $X$. Also $(X, c)$ is called $L$ -closure space.

Definition 2.5. In an $L$-closure space $(X, c)$, an $L$-subset $f$ of $X$ is said to be $L$ -closed if $c(f)=f$. An L-subset $f$ of $X$ is L-open if its complement is closed in $(X, c)$. 
A Note on the Lattice of $L$-Closure Operators

The set of all open $L$-subsets of $(X, c)$ forms an $L$-topology on $X$, called the $L$ -topology associated with the $L$-closure operator $c$.

Let $F$ be an $L$-topology on a set $X$. Then a function $c: L^{X} \rightarrow L^{X}$ defined by $c(f)=\bar{f}$ for all $f \in L^{X}$, where $\bar{f}$ is the closure of $f$ in $\left(L^{X}, F\right)$, is an $L$-closure operator on $X$ called the $L$-closure operator associated with the $L$-topology $F$.

An $L$-closure operator on a set $X$ is called $L$-topological if it is the $L$-closure operator associated with an $L$-topology on $X$.

Remark 2.6. Note that the different $L$-closure operators can have the same associated $L$-topology. But different $L$-topologies can not have the same associated $L$-closure operator.

Example 2.7. Let $X=\{x, y, z\}$ and $L=\{0, \alpha, \beta, 1\}$ be a chain with $0<\alpha<\beta<1$. Then the functions $c_{1}, c_{2}: L^{X} \rightarrow L^{X}$ defined by:

$$
c_{1}(f)=\left\{\begin{array}{llc}
\underline{0} & \text { if } & f=\underline{0} \\
g & \text { if } & f=x_{\alpha} \\
\underline{1} & & \text { otherwise }
\end{array},\right.
$$

where $g \in L^{X}$ is defined as $g(x)=g(y)=1$ and $g(z)=\beta$

$$
\text { and } c_{2}(f)=\left\{\begin{array}{llc}
\underline{0} & \text { if } & f=\underline{0} \\
\underline{1} & \text { otherwise }
\end{array}\right.
$$

are $L$-closure operators. Associated $L$-topologies of $c_{1}$ and $c_{2}$ are same, which is the indiscrete $L$-topology.

\section{Lattice of $L$-closure operators}

Definition 3.1. Let $c_{1}$ and $c_{2}$ be $L$-closure operators on $X$. Then $c_{1} \leq c_{2}$ if and only if $c_{2}(f) \leq c_{1}(f), \forall f \in L^{X}$.

Remark 3.2. The set $L C(X)$ of all $L$-closure operators forms a lattice with this relation $\leq$. If $c_{1}, c_{2} \in L C(x)$, then the join $c_{1} \vee c_{2}$ and the meet $c_{1} \wedge c_{2}$ are defined respectively by the following formulas :

$\left(c_{1} \vee c_{2}\right)(x)=\min \left\{c_{1}(x), c_{2}(x)\right\}$ and

$\left(c_{1} \wedge c_{2}\right)(x)=\max \left\{c_{1}(x), c_{2}(x)\right\}$.

Definition 3.3. The $L$-closure operator $D$ defined on $X$ by $D(f)=f$ for all $f \in L^{X}$, is called the discrete $L$-closure operator.

The $L$-closure operator $I$ on $X$ defined by

$$
I(f)=\left\{\begin{array}{llr}
\underline{0} & \text { if } & f=\underline{0} \\
\underline{1} & & \text { otherwise }
\end{array},\right.
$$




\section{Pinky and T.P Johnson}

is called the indiscrete $L$-closure operator.

Remark 3.4. $D$ and $I$ are the $L$-closure operators associated with the discrete and indiscrete $L$-topologies on $X$ respectively. Moreover $D$ is the unique $L$-closure operator whose associated L-topology is discrete. Also $I$ and $D$ are the smallest and the largest elements of the lattice $L C(X)$ respectively.

Theorem 3.5. [5] $L C(X)$ is a complete lattice.

In [8], we find the following theorem:

Theorem 3.6. [8] $L C(X)$ is not modular.

But this result is not true as shown by the following theorem:

Theorem 3.7. $L C(X)$ is a distributive lattice.

Proof: Let $c_{1}, c_{2}$ and $c_{3}$ be any three elements of $L C(X)$.

Then by definition of $\leq$, we have

$c_{1} \vee\left(c_{2} \wedge c_{3}\right)=\min \left[c_{1}, \max \left\{c_{2}, c_{3}\right\}\right]$

and $\left(c_{1} \vee c_{2}\right) \wedge\left(c_{1} \vee c_{3}\right)=\max \left[\min \left\{c_{1}, c_{2}\right\}, \min \left\{c_{1}, c_{3}\right\}\right]$.

For any $f \in L^{X}$ and $x \in X$, assume that $c_{1}(f)=g_{1}, c_{2}(f)=g_{2}, c_{3}(f)=g_{3}$ and $g_{1}(x)=\alpha, g_{2}(x)=\beta, g_{3}(x)=\gamma$.

Since $\alpha, \beta, \gamma \in L$ and $L$ is a chain, the following six case arise:
(1) $\gamma<\beta<\alpha$
(2) $\beta<\gamma<\alpha$
(3) $\alpha<\gamma<\beta$
(4) $\gamma<\alpha<\beta$
(5) $\beta<\alpha<\gamma \quad$ (6)

$\alpha<\beta<\gamma$.

Case 1: $\gamma<\beta<\alpha$.

Then $\left\{c_{1} \vee\left(c_{2} \wedge c_{3}\right)\right\}(f)(x)$

$=\min \left\{g_{1}(x), \max \left\{g_{2}(x), g_{3}(x)\right\}\right\}$

$=\min \{\alpha, \max \{\beta, \gamma\}\}$

$=\min \{\alpha, \beta\}=\beta$

and $\left\{\left(c_{1} \vee c_{2}\right) \wedge\left(c_{1} \vee c_{3}\right)\right\}(f)(x)$

$=\max \left\{\min \left\{g_{1}(x), g_{2}(x)\right\}, \min \left\{g_{1}(x), g_{3}(x)\right\}\right\}$.

$=\max \{\min \{\alpha, \beta\}, \min \{\alpha, \gamma\}\}$.

$=\max \{\beta, \gamma\}=\beta$.

In the same way, it can be checked that the equality

$\left\{c_{1} \vee\left(c_{2} \wedge c_{3}\right)\right\}(f)(x)=\left\{\left(c_{1} \vee c_{2}\right) \wedge\left(c_{1} \vee c_{3}\right)\right\}(f)(x)$ holds good in the remaining five cases also.

Since $f \in L^{X}$ and $x \in X$ were arbitrary, 
A Note on the Lattice of $L$-Closure Operators

$\Rightarrow c_{1} \vee\left(c_{2} \wedge c_{3}\right)=\left(c_{1} \vee c_{2}\right) \wedge\left(c_{1} \vee c_{3}\right)$.

$\Rightarrow L C(X)$ is distributive lattice.

Corollary 1. $L C(X)$ is a modular lattice.

\section{Infra $L$-closure operators}

Definition 4.1. An L-closure operator on $X$ is called an infra $L$-closure operator if the only $L$-closure operator on $X$ strictly smaller than it is $I$.

Theorem 4.2. [5] If $L=[0,1]$, then there is no infra $L$-closure operator in $L C(X)$.

In [8], we find the following result :

Let $X$ be any set and $a, b \in X$ such that $a \neq b$. Define $\psi_{a, b}: L^{X} \rightarrow L^{X}$ by

$$
\psi_{a, b}(f)=\left\{\begin{array}{ccc}
f & \text { if } & f=\underline{0} \\
g_{\alpha, b} & \text { if } & f=a_{\alpha} \\
\underline{1} & & \text { otherwise }
\end{array},\right.
$$

where $\alpha$ is a dual atom in $L$ and $g_{\alpha, b}$ is defined by

$$
g_{\alpha, b}(a)=\left\{\begin{array}{lll}
1 & \text { if } & a \neq b \\
\alpha & \text { if } & a=b
\end{array} .\right.
$$

Theorem 4.3. [8] An L-closure operator is an infra $L$-closure operator if and only if it is of the form $\psi_{a, b}$ for some $a, b \in X, a \neq b$.

But this result is not true because $\psi_{a, b}$ is not even an $L$-closure operator as shown below:

Let $a_{\alpha}, a_{\eta} \in L^{X}$, where $\eta, \alpha \in L$ such that $\eta<\alpha$ and $\alpha$ is a dual atom in $L$.

Then $\psi_{a, b}\left(a_{\alpha} \vee a_{\eta}\right)=\psi_{a, b}\left(a_{\alpha}\right)=g_{\alpha, b}$

and $\psi_{a, b}\left(a_{\alpha}\right) \vee \psi_{a, b}\left(a_{\eta}\right)=g_{\alpha, b} \vee \underline{1}=\underline{1}$.

$\Rightarrow \psi_{a, b}\left(a_{\alpha} \vee a_{\eta}\right) \neq \psi_{a, b}\left(a_{\alpha}\right) \vee \psi_{a, b}\left(a_{\eta}\right)$.

Remark 4.4. Let $X$ be any nonempty set and $L$ be a finite chain with the atom $\alpha$ and the dual atom $\beta$. For any $x, y \in X$, define $c_{x, y}: L^{X} \rightarrow L^{X}$ by

$$
c_{x, y}(f)=\left\{\begin{array}{ccc}
\underline{0} & \text { if } & f=\underline{0} \\
g_{y, \beta} & \text { if } & f=x_{\alpha} \\
\underline{1} & & \text { otherwise }
\end{array},\right.
$$

where $g_{y, \beta} \in L^{X}$ is defined as $g_{y, \beta}(y)=\beta$ and $g_{y, \beta}(z)=1, \forall z(\neq y) \in X$. Clearly, 


\section{Pinky and T.P Johnson}

$g_{y, \beta}$ is a dual atom in $L^{X}$.

It can be easily checked that $c_{x, y}$ is an $L$-closure operator and $g_{y, \beta}$ can be replaced by any dual atom in $L^{X}$. Therefore the number of such $L$-closure operators is $|X|^{2}$.

Theorem 4.5. Let $X$ be a nonempty set and $L$ be a chain with the atom $\alpha$ and the dual atom $\beta$. An L-closure operator is an infra $L$-closure operator if and only if it is of the form $c_{x, y}$ for some $x, y \in X$.

Proof: Let $c$ be any $L$-closure operator on $X$ such that

$$
c \leq c_{x, y} \Rightarrow c_{x, y}(f) \leq c(f), \forall f \in L^{X} .
$$

Therefore $c(f)=\underline{1}, \forall f\left(\neq x_{\alpha}\right) \in L^{X}$ and $g_{y, \beta} \leq c\left(x_{\alpha}\right)$. Since $g_{y, \beta}$ is a dual atom in $L^{X}$, it follows that either $c\left(x_{\alpha}\right)=g_{y, \beta}$ or $c\left(x_{\alpha}\right)=\underline{1}$.

If $c\left(x_{\alpha}\right)=g_{y, \beta}$, then $c=c_{x, y}$ and if $c\left(x_{\alpha}\right)=\underline{1}$, then $c=I$.

Hence $c_{x, y}$ is an infra $L$-closure operator.

Conversely, suppose that $c$ is any infra $L$-closure operator in $L C(X)$. Then $c$ must be of the form

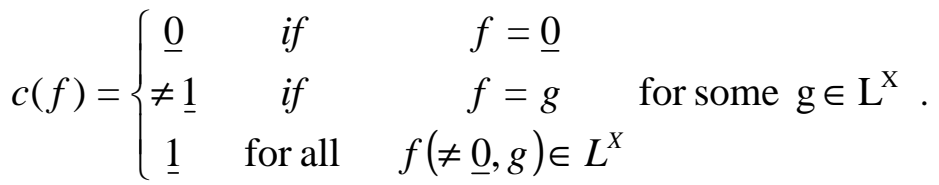

If $\exists$ an $L$-subset $h(\neq \underline{0}) \in L^{X}$ such that $h<g$, then $h \vee g=g$ and $c(h \vee g)=c(g)$.

$\Rightarrow c(h) \vee c(g)=c(g)$

$\Rightarrow \underline{1} \vee c(g)=c(g)$

$\Rightarrow c(g)=\underline{1}$, a contradiction.

$\Rightarrow g$ must be an atom in $L^{X}$ i.e. $g=x_{\alpha}$ for some $x \in X$ and for the atom $\alpha \in L$. Now, if $c(g)<f$ for some $f(\neq \underline{1}) \in L^{X}$, then $c_{1}: L^{X} \rightarrow L^{X}$ defined by :

$$
c_{1}(h)=\left\{\begin{array}{llc}
\underline{0} & \text { if } & h=\underline{0} \\
f & \text { if } & h=x_{\alpha} \\
\underline{1} & & \text { otherwise }
\end{array}\right.
$$

is an $L$-closure operator such that $c_{1} \neq I$ and $c_{1}<c$, a contradiction. Therefore $c(g)$ must be a dual atom in $L^{X}$ i.e. $c(g)(t)=\underline{1}, \forall t \in X$ except for some $y \in X$ and $c(g)(y)=\beta . \Rightarrow c=c_{x, y}$.

Thus all infra $L$-closure operators are of the form $c_{x, y}$ for some $x, y \in X$. 
A Note on the Lattice of $L$-Closure Operators

Remark 4.6. If $L$ is a chain with the atom $\alpha$ and the dual atom $\beta$, then for any nonempty set $X$, there are $|X|^{2}$ infra $L$-closure operators in $L C(X)$.

Remark 4.7. Let $X=\{x, y, z\}$ and $L=\{0, \alpha, \beta, 1\}$ be a chain with $0<\alpha<\beta<1$. Then there are 9 infra $L$-closure operators given by

$$
c_{x, y}(f)=\left\{\begin{array}{ccc}
\underline{0} & \text { if } & f=\underline{0} \\
g_{y, \beta} & \text { if } & f=x_{\alpha} \\
\underline{1} & & \text { otherwise }
\end{array}\right.
$$

where $x, y \in X$ and $g_{y, \beta} \in L^{X}$ is defined as $g_{y, \beta}(y)=\beta$ and $g_{y, \beta}(x)=g_{y, \beta}(z)=1$ . It can be easily checked that the $L$-closure operator $c: L^{X} \rightarrow L^{X}$ defined by

$$
c(f)=\left\{\begin{array}{llc}
\underline{0} & \text { if } & f=\underline{0} \\
\underline{\alpha} & \text { if } & f=x_{\beta} \\
\underline{1} & & \text { otherwise }
\end{array}\right.
$$

can not be written as the join of infra $L$-closure operators. Thus in general $L C(X)$ is not an atomic lattice.

\section{Ultra $L$-closure operators}

Definition 5.1. An L-topology $F$ on $X$ is called an ultra $L$-topology if the only $L$ -topology on $X$ strictly finer than $F$ is the discrete $L$-topology.

Definition 5.2. An $L$-closure operator on $X$ is called an ultra $L$-closure operator if the only $L$-closure operator on $X$ strictly larger than it, is $D$.

Theorem 5.3. Let $c_{1}$ and $c_{2}$ be two $L$-closure operators such that $c_{1} \leq c_{2}$. If $\mathrm{F}_{1}$ and $F_{2}$ are the $L$-topologies associated with the $L$-closure operators $c_{1}$ and $c_{2}$ respectively, then $\mathrm{F}_{1} \subseteq \mathrm{F}_{2}$.

Proof: Let $g \in \mathrm{F}_{1}$.

$\Rightarrow c_{1}\left(g^{\prime}\right)=g^{\prime}$, where $g^{\prime}$ is complement of $g$.

$\Rightarrow g^{\prime} \leq c_{2}\left(g^{\prime}\right) \leq c_{1}\left(g^{\prime}\right)=g^{\prime}$.

$\Rightarrow c_{2}\left(g^{\prime}\right)=g^{\prime}$.

$\Rightarrow g \in \mathrm{F}_{2} \Rightarrow \mathrm{F}_{1} \subseteq \mathrm{F}_{2}$.

Theorem 5.4. Let $\mathrm{F}_{1}$ and $\mathrm{F}_{2}$ be two L-topologies such that $\mathrm{F}_{1} \subseteq \mathrm{F}_{2}$. If $c_{1}$ and $c_{2}$ are the $L$-closure operators associated with the L-topologies $F_{1}$ and $F_{2}$ respectively, then $c_{1} \leq c_{2}$.

Proof: For any $f \in L^{X}$, let $\bar{f}_{1}$ and $\bar{f}_{2}$ be the closure of $f$ in the $L$-topological 
Pinky and T.P Johnson

spaces $\left(L^{X}, \mathrm{~F}_{1}\right)$ and $\left(L^{X}, \mathrm{~F}_{2}\right)$ respectively.

Since $\mathrm{F}_{1} \subseteq \mathrm{F}_{2}$,

$\Rightarrow \bar{f}_{2} \leq \bar{f}_{1}$.

$\Rightarrow c_{2}(f) \leq c_{1}(f)$.

$\Rightarrow c_{1} \leq c_{2}$.

Theorem 5.5. Let $c$ be an ultra $L$-closure operator. If $\mathrm{F}$ is the $L$-topology associated with the $L$-closure operator $c$, then $\mathrm{F}$ is an ultra $L$-topology.

Proof: If $F$ is not an ultra $L$-topology, then there exists an $L$-topology $F_{1}$ such that $\mathrm{F}_{1} \neq L^{X}$ and $\mathrm{F} \subset \mathrm{F}_{1}$. Let $c_{1}$ be the $L$-closure operator associated with the $L$-topology $\mathrm{F}_{1}$. Then $c_{1} \neq D$.

Since $\mathrm{F} \subset \mathrm{F}_{1}$,

$\Rightarrow c \leq c_{1}$ and $\exists$ an $L$-subset $g \in L^{X}$ such that $g \in \mathrm{F}_{1}$ but $g \notin \mathrm{F}$.

$\Rightarrow c_{1}\left(g^{\prime}\right)=g^{\prime}$ and $c\left(g^{\prime}\right) \neq g^{\prime}$

$\Rightarrow c<c_{1}$, a contradiction.

Hence $F$ is an ultra $L$-topology.

Theorem 5.6. Let $\mathrm{F}$ be an ultra $L$-topology. If $c$ is the $L$-closure operator associated with the $L$-topology $\mathrm{F}$, then $c$ is an ultra $L$-closure operator.

Proof: Suppose, there exists an $L$-closure operator $c_{1}$ such that $c \leq c_{1}$. Let $\mathrm{F}_{1}$ be the $L$-topology associated with the $L$-closure operator $c_{1}$.

Since $c \leq c_{1} \Rightarrow \mathrm{F} \subseteq \mathrm{F}_{1}$ and $\mathrm{F}$ is an ultra $L$-topology so it follows that either $\mathrm{F}_{1}=\mathrm{F}$ or $\mathrm{F}_{1}=L^{X}=$ discrete $L$-topology.

If $\mathrm{F}_{1}=\mathrm{F}$, then $c_{1}=c$ and if $\mathrm{F}_{1}=L^{X}$, then $c_{1}=D=$ discrete $L$-closure operator.

Hence $c$ is an ultra $L$-closure operator.

Theorem 5.7. Let $X$ be a nonempty set and $L$ be a bounded chain. Then an $L$ -closure operator is an ultra $L$-closure operator if and only if it is the $L$-closure operator associated with some ultra $L$-topology on $X$.

Remark 5.8. If $L=[0,1]$, then there is no ultra $L$-topology in $L^{X}$ and hence no ultra $L$-closure operator in $L C(X)[4]$.

Remark 5.9. Let $X=\{x, y, z\}$ and $L=\{0, \alpha, \beta, 1\}$ be a chain with $0<\alpha<\beta<1$.

Then the $L$-closure operator $c: L^{X} \rightarrow L^{X}$ defined by 
A Note on the Lattice of $L$-Closure Operators

$$
c(f)=\left\{\begin{array}{llc}
\underline{0} & \text { if } & f=\underline{0} \\
\underline{\alpha} & \text { if } & f=x_{\beta} \\
\underline{1} & & \text { otherwise }
\end{array}\right.
$$

has no complement $\Rightarrow L C(X)$ is not complemented in general.

Remark 5.10. [5] If $L=[0,1]$, then $L C(X)$ is not complemented.

\section{Conclusion}

In this paper, we have identified infra $L$-closure operators and established a relation between ultra $L$-closure operators and ultra $L$-topologies. Also it is proved that $L C(X)$ is a distributive lattice when $L$ is a bounded chain. Lattice of $L$-closure operators when $L$ is a bounded lattice other than a chain, will be discussed in future papers.

Acknowledgement. The first author wishes to thank the University Grants Commission, India, for giving financial support.

The authors are thankful to the referees for their valuable suggestions.

\section{REFERENCES}

1. E Cech, Topological Spaces, Interscience Publishers, John Wiley and Sons New York, 1996.

2. C.L.Chang, Fuzzy topological spaces, Journal of Mathematical Analysis and Applications, 24 (1968) 182-190.

3. B.A.Davey, H.A.Priestley, Introduction to Lattices and Order, second edition, Cambridge University Press, New York, 2002.

4. T.P.Johnson, On the lattice of fuzzy topologies I, Fuzzy Sets and Systems, 48 (1992) 133-135.

5. T.P.Johnson, Completely homogeneous fuzzy closure spaces and lattice of fuzzy closure operators, Fuzzy Sets and Systems, 52 (1992) 89-91.

6. Y.-M. Liu, M.-K. Luo, Fuzzy Topology, World Scientific Publishing Co., River Edge, New Jersey, 1997.

7. A.S.Mashhour and M.H.Ghanim, Fuzzy closure spaces, Journal of Mathematical Analysis and Applications, 106(1) (1985) 154-170.

8. R.George , T.P.Johnson, On the Lattice of $L$-closure Operators, International Journal of Science and Research, 2(3) (2013) 409-412.

9. R.Srivastava, A.K.Srivastava and A.Choubey, Fuzzy Closure spaces, Journal of Fuzzy Mathematics, 2 (1994) 525-534.

10. R.Srivastava and M.Srivastava, On $\mathrm{T}_{0}$ and $\mathrm{T}_{1}$ fuzzy closure spaces, Fuzzy Sets and Systems, 109(2) (2000) 263-269.

11. W.-N.Zhou, Generalization of L-closure spaces, Fuzzy Sets and Systems, 149(3) (2005) 415-432.

12. L.A.Zadeh, Fuzzy sets, Inform and Control, 8 (1965) 338-353. 